\title{
Role of gut microbiota in dog and cat's health and diseases
}

\author{
Elisabetta Mondo*, Giovanna Marliani, Pier Attilio Accorsi, Massimo Cocchi, and Alberto Di Leone
}

Department of Veterinary Medical Sciences, University of Bologna, Bologna, Italy

\begin{abstract}
Mammalian gastrointestinal tract is colonized by a large number of microorganisms, known as gut microbiota, that play a key role in the physiological and pathological states. In particular, the gastrointestinal tract of dogs and cats harbors a complex and highly biodiverse microbial ecosystem. Recent studies see it involved in a wide range of life processes, including energy needs, metabolism, immunological activity, and neuro-behavioral development. This review focuses on the role of the microbiota on the health of pets and will discuss changes that occur in the disease. Keywords: Cat, Dog, Gut, Microbiota.
\end{abstract}

\section{Introduction}

Bacteria, archaea, fungi, protozoa, and viruses, which have a positive role on life processes and host health, inhabit the gastrointestinal tract (GI) of cats and dogs (Suchodolski, 2011). Different body sites present their own microbiota and gut microbiota is the largest one. It is composed by $10^{13}-10^{14}$ cells (more than 1,000 bacteria species) and its genome is 150 times larger than most of animals' genome (Simpson et al., 2002). Depending on $\mathrm{pH}$ value and residence-time, there is a different concentration of bacteria in different GI tracts (Savage, 1977). Recently, the development of new molecular technologies, such as next-generation sequencing (NGS), has allowed understanding the complexity and diversity of gut-microbial communities (Junhyung et al., 2017). Molecular-phylogenetic analysis of the bacterial $16 \mathrm{~S}$ rRNA gene has created a more detailed inventory of bacteria groups present in the bowel. Although there are differences of GI inter- and intraspecies, 16S rRNA gene sequencing has shown that more than $90 \%$ of bacteria belong to two phyla: Bacteroidetes and Firmicutes (Simpson et al., 2002; Ritchie et al., 2008). The phylum Firmicutes includes Clostridium clusters, which include Ruminococcus spp., Faecalibacterium spp., and Dorea spp. These bacteria, Bacteroidetes and Actinobacteria can produce short-chain fatty acids (SCFAs), the essential energy source for colonocytes. Moreover, they help to maintain the epithelial barrier by strengthening tight junctions, to regulate intestinal motility and to stimulate the production of anti-inflammatory compounds. Because of its important functions, the gut microbiota is frequently defined as "forgotten organ" (O'Hara and Shanahan, 2006).

Many research studies on mammalians have highlighted how the microbiota is involved inmany hostlife-processes, such as energy requirement, metabolism, immunologic activity, and neuro-behavioral development. Changes in gut-microbiome are associated with diseases, including inflammation, obesity, metabolic syndromes, and mood disorders. A balanced microbial ecosystem is crucial for host health and homeostasis (Guard et al., 2017).

Role of the physiological gut microbiota

The interaction between gut microbiota, its host, and other somatic cells regulates many functions, such as digestion, host metabolism, vitamins synthesis (vit. K and complex B), biotransformation of bile acids, xenobiotics metabolism, correct maturation of gastrointestinal cells, and defense against pathogenic bacteria (Steiner and Ruaux, 2008). Therefore, the microbiota can be defined as a metabolically active "organ."

Genetic diversity of microbiota in the gut guarantees the presence of many enzymes and biochemical pathways that otherwise the host does not possess.

The bacteria metabolic activities produce energy and substrates useful both to bacteria for their proliferation and to the host. The main activity is the fermentation of alcohols and non-digestible carbohydrates (starch, inulin, cellulose, hemicellulose, pectin, and gum) (Schmitz and Suchodolski, 2016). It occurs in the colon (Bell et al., 2008) and it causes the production of gasses and SCFAs, acetate, propionate, and butyrate (60:25:15), which give energy to the intestinal epithelium and other tissues (Wong et al., 2006). SCFAs synthesis leads to a reduction of intestinal $\mathrm{pH}$ value, which is the first sensory line of defense and it supports growth, proliferation, and differentiation of epithelial intestinal cells. Commensal bacteria defend the host from pathogens, producing bacteriocins and colicins and competing for nutrients and ecological benefits (Liévin-Le Moal and Servin, 2006). Moreover, when SCFAs are absorbed through the intestine, they allow the re-absorption of $\mathrm{Na}+$ or $\mathrm{K}+$ ions (Fleming et al., 1991).

Another important role of microbiota is the transformation both of primary bile acids (Colic acid and chenodeoxycholic acid) and secondary bile acids

*Corresponding Author: Elisabetta Mondo. Department of Veterinary Medical Sciences, University of Bologna, via Tolara di Sopra 50, 40064, Ozzano dell’Emilia, Bologna, Italy. Email: elisabetta.mondo2@unibo.it 
(deoxycholic and lithocholic), which are essential in the absorption of dietary fats and liposoluble vitamins in the gut (Staggers et al., 1982).

Furthermore, commensal bacteria have a fundamental role on the induction, shaping, and function of the host immune system, which in turn is important in the development of the physiological gut structure and the identification of pathogens from commensal bacteria. This relationship allows the defensive responses against pathogens being effective and it regulates the pathways involved in the tolerance of innocuous antigens. The ability of microbes to set the immunological tone of tissues, both locally and systemically, requires tonic sensing of microbes and complex feedback loops between innate and adaptive components of the immune system (Suchodolski and Simpson, 2013). The intestinal mucosa is an interface between the immune system and the external environment. Results of several studies have shown that germ-free animals have a lower density of lymphoid cells in the intestinal mucosa and a lower quantity of immunoglobulins (Ig) in serum. The exposure of these animals to commensal microbes induces a rapid increase of lymphocytes and Ig in the bowel and of Ig in the serum (Capurso, 2016). Development and regulation of lymphocytes $T$ helper type 1 and 2 are influenced by bacteria, which can suppress NF-kB through the production of butyrate and acetate and can act on the receptors linked G-protein. There is a dynamic relationship between the immune system and microbiota. Intestinal mucosa, with Tolllike receptors, induces fall signal and provokes the production and the release of inflammatory mediators (cytokines, phagocytes) (Schmitz and Suchodolski, 2016; Garraway et al., 2018).

\section{Dysbiosis of the gut microbiota in animal disease}

Hippocrates used to say: "bad digestion is the root of all evil." This sentence has held up to modern scientific scrutiny.

As previously described, the microbiome participates in the vital physiologic and immunologic process, but it is unclear how it directly affects the pathogenesis of a disease state.

In healthy conditions, the crosstalk and the crossregulation between the host and the microorganisms create a homeostatic balance of bacteria, so the gastrointestinal tract remains healthy and free from an overgrowth of potentially pathogenic bacteria. In that case, there is a state of eubiosis. When this condition of homeostasis is disrupted, dysbiosis occurs. In case of dysbiosis, there is an imbalance in bacterial composition, and bacterial metabolic activities and/or bacterial distribution inside the gut change. Three types of dysbiosis can be defined as follows:

- reduction of bacterial diversity;

- loss of beneficial bacteria;

- overgrowth of pathogens.
Each of those cases is linked to other problems (Xenoulis et al., 2008). A state of dysbiosis is found in a wide range of diseases, such as inflammatory bowel disease (IBD), obesity, allergy, diabetes, and autism, but it is unclear if it is a cause or a consequence. Several studies about these diseases have indicated the presence of a microbial alteration, but no consistent pattern of microbiota changes has yet been observed. In IBD dogs, Minamoto et al. (2014) have highlighted an increase of Gammaproteobacteria and a decrease of Erysipelotrichia, Clostridia, and Bacteroidia, using 454-pyrosequencing on fecal samples. Suchodolski et al. (2012) had applied the same method on the same kind of samples and they found IBD dogs characterized by an increase in Sutterella and Clostridium perfringens, and a decrease in Blautia, Ruminococcaceae, and Turicibacter. Even if the result of the two studies is not the same, they both indicate that a reduction in the diversity of Clostridium clusters XIVa and IV in dog gut microbiota are associated with IBD. The same condition has been found in human and cats IBD. These results underline the importance of those clusters of bacteria, which also produce SCFAs, in the maintenance of gastrointestinal health (Suchodolski et al., 2012; Garcia-Mazcorro and Minamoto, 2013).

Manipulation of the gastrointestinal microbiome

During a state of disease, therapies applied have the purpose to change the microbiota community. Besides the diet, probiotics, prebiotics and antibiotics administration affect, and change microbiota composition, but their efficiency is not clear. Recently, it has been developed a new method that considers restoration of the colonic microflora by introducing healthy bacterial flora. It is named fecal microbiota transplantation (FMT) (Cammarota et al., 2014).

The following paragraphs are going to describe the different strategies applied and the results of the recent research studies.

\section{Prebiotics and probiotics}

Use of pre- and probiotic is broadly spread in human medicine to preserve or restore a healthy condition (Sanders et al., 2018). The employment of these devices is new in veterinary medicine and pet treatment.

Prebiotics are more recent and, in accordance to their first definition given in 2015, they are "a nondigestible compound that, through its metabolization by microorganisms in the gut, modulates composition and/or activity of the gut microbiota, thus conferring a beneficial physiological effect on the host" (Bindels et al., 2015).

Nowadays, several research studies report benefits from the addition of prebiotics in pets' diet. Indeed, they beneficially modulate gut microbiota and protect the animals from enteric infections.

There are some studies about their use in the domestic canine diet. Zentek et al. (2003) found that in dogs, an intake of $1.5 \%$ inulin could reduce fecal $\mathrm{pH}$ and increase Bifidobacteria population. Middelbos et al.(2010), using 
16S rRNA sequencing, have shown that dogs fed with relatively small amount of dietary fiber change structure of gut microbiota, increasing the density of Firmicutes and decreasing that of Fusobacteria. Another study underlined how a dietary supplementation of fructooligosaccharides (FOS) induces beneficial effects, such as the growth of Bifidobacteria, and it improves the digestibility of several minerals in the entire GI tract in the dog (Pinna et al., 2018).

Few research studies about feline nutrition and use of prebiotics have been conducted. Two studies describe that the high-protein dietary intake, typical of cat diet, mainly induces the growth of fecal Clostridium (Lubbs et al., 2009; Hooda et al., 2013). Barry et al. (2010) have found that the addition of FOS to feline diet bring to an increase of Bifidobacteria concentration and to a decrease of Escherichia coli population. The same research showed how the addition of pectin in cat nutrition raises the $C$. perfringens and lactobacilli concentration (Barry et al., 2010). Table 1 shows a summary of the studies on the effect of prebiotics on dog and cat.

Thousands of years ago, human beings discovered benefits deriving from fermented food (Di Gioia and Biavati, 2018). Therefore, the employment of probiotic is more ancient than that of prebiotic. They are defined as "live microorganisms that when administered in adequate amounts confer a health benefit on the host" (FAO/WHO, 2001). Both in human and pets, probiotics have many beneficial effects on host health, especially in the modulation of the immune system and in stress state. The use of probiotics in livestock is broadly spread, whereas in pet nutrition is still developing (Di Gioia and Biavati, 2018).

Sauter et al. (2005) conducted an ex vivo study in dogs with chronic enteropathies. This research confirmed the positive effect of a probiotic cocktail, containing three different Lactobacillus strains, on cytokine expression, mainly through the regulation of T-cells. Rossi et al.(2014) found that the administration of probiotics promotes the intensification of T-cell expression. In this study, a probiotic cocktail, similar to that of human VSL\#3, was formulated for dogs with IBD. Data highlighted an increase of T-cell's target, a decrease of Faecalibacterium population, and recovery from the dysbiosis.

From weaning to 1 year of age, puppies' diet is often supplemented through addition of probiotics. Indeed, it has been demonstrated the presence of adjuvant effects of Enterococcum faecium at both intestinal mucosal level and systemic level. It is possible that these effects are relevant in the improvement of the protective immune response during the weaning period (Benyacoub et al., 2003).

Table 2 shows a summary of the studies on the effect of prebiotics treatment.

\section{Antibiotics}

Dogs and cats with chronic enteropathies are treated with antibiotics when diet change does not work (Makielski et al., 2018). The most common molecules

Table 1. Studies of prebiotic treatment in cat and dog.

\begin{tabular}{|c|c|c|c|}
\hline Animal & Prebiotic treatment & Effect of treatment & References \\
\hline Cat & $\begin{array}{l}\text { Diet with the supplement of FOS and } \\
\text { cellulose }\end{array}$ & $\begin{array}{c}\text { Increase of Bifidobacteria and decrease of } \\
\text { Escherichia coli }\end{array}$ & Barry et al., 2010 \\
\hline Cat & $\begin{array}{l}\text { Diet with the supplement of FOS and } \\
\text { pectin }\end{array}$ & $\begin{array}{l}\text { Increase of lactobacilli and decrease of } \\
\text { Clostridium perfringens }\end{array}$ & Barry et al., 2010 \\
\hline Cat & Diet with high intake protein & Increase fecal Clostridium & Lubbs et al., 2009 \\
\hline Dog & $\begin{array}{l}\text { Dry diets, one supplemented with } 3 \% \\
\text { chicory }(1.5 \% \text { inulin) }\end{array}$ & $\begin{array}{l}\text { Reduction of fecal } \mathrm{pH} \text { and increase } \\
\text { Bifidobacteria population }\end{array}$ & Zentek et al., 2003 \\
\hline Dog & $\begin{array}{c}\text { diet with } 7.5 \% \text { beet pulp ( } 60 \% \text { total } \\
\text { dietary fiber, } \sim 4: 1 \text { insoluble:soluble fiber) }\end{array}$ & $\begin{array}{l}\text { Increase the density of Firmicutes and } \\
\text { decrease that of Fusobacteria }\end{array}$ & Middelbos et al., 2010 \\
\hline Dog & Diet with the supplement of FOS & Growth of Bifidobacteria & Pinna et al., 2018 \\
\hline
\end{tabular}

Table 2. Summary of the studies on the effect of probiotics treatment.

\begin{tabular}{ccccc}
\hline Animal & Disease & Probiotic treatment & Effect of treatment & References \\
\hline Dog & $\begin{array}{c}\text { Chronic } \\
\text { enteropathies }\end{array}$ & $\begin{array}{c}\text { Administration of mixture of probiotic } \\
\text { bacteria: Lactobacillus spp. (two } \\
\text { Lactobacillus acidophilus strains } \\
\text { (NCC2628, NCC2766), one Lactobacillus } \\
\text { johnsonit strain (NCC2767) }\end{array}$ & $\begin{array}{c}\text { Beneficial effects on } \\
\text { cytokine expression } \\
\text { mainly through regulatory } \\
\text { T-cells }\end{array}$ & $\begin{array}{c}\text { Sauter } \text { et al., 2005 } \\
\text { Dog }\end{array}$ \\
& IBD & Administration of VSL\#3 & $\begin{array}{c}\text { Significant decrease in } \\
\text { clinical and histological } \\
\text { scores and decrease in }\end{array}$ & Rossi et al., 2014 \\
& & & CD3+ T-cell infiltration. & \\
\hline
\end{tabular}


used are metronidazole and tylosin (Simpson, 2011). Metronidazole acts on bacteria and protozoa. Usually, it is used simultaneously to nutritional therapy and a diet change, so it is difficult to know its real effect (Jergens et al., 2003; Craven et al., 2004; Münster et al., 2006; Garcìa-Sancho et al., 2007).

Tylosin is employed in the treatment of tylosinresponsive chronic diarrhea, which usually affects adult dogs. Many studies underline its efficiency (Westermarck et al., 2005; Kilpinen et al., 2011) but its mechanism of action is still unknown.

Enrofloxacin is another antibiotic used in enteropathies. It is a fluoroquinolone useful in the therapy of granulomatous colitis of Boxer (Davies et al., 2004).

The use of antibiotics in the treatment of certain diseases has contraindications. Indeed, abundant employment of antibiotics may reduce beneficial bacteria population, facilitate the increase of potential pathogens population, and promote the phenomenon of antimicrobial resistance. Some researchers have shown that antibiotics actually modify the microbial composition decreasing bacteria diversity, so they contribute to the onset of dysbiosis (Grønvold et al., 2010). Over the past years, the phenomenon of antimicrobial resistance has been increasing and has assumed a great importance. The close cohabitation of human with pets can cause the transfer of antimicrobial resistance to bacteria that affect also humans (Damborg et al., 2008).

\section{Fecal microbiota transplantation (FMT)}

FMT is a medical non-pharmacological experimental treatment, which aims to restore microbial diversity. It consists in a procedure of transplant of fecal matter from a healthy donor to a patient affected by a gastrointestinal disease that does not respond to common therapies. It is made using colonoscopy, endoscopy, sigmoidoscopy, or enema (Cammarota et al., 2014). Fecal transplant was an ancient procedure, firstly documented in the 4th century China. However, it has gained popularity during recent years. In human medicine, this procedure is used in the therapy of Clostridium difficile infection, with an efficiency of $87 \%-90 \%$ (Bakken et al., 2011), and in the treatment of IBD (Kelly et al., 2015).

Research studies about the FMT application in veterinary medicine are few, even if they dare are promising. This treatment has been employed in dogs affected by gastrointestinal disease not respondent to common therapy, such as IBD and Clostridium perfringes infection. A recent case report describes the use of FMT in the treatment of one dog with refractory IBD and one cat with non-responsive chronic enteropathy. In both cases, fecal consistency is improved within 24 hours post FMT (Weese et al., 2001).

Even if FMT seems to be relatively sure and an efficient therapy for the treatment of dysbiosis, more clinical research studies are necessary to understand better its mechanisms and potential risks for the patients that receive these infusions. Nowadays, only limited data are available.

\section{Conclusion}

The gut microbiota is essential for the health of all mammals because it participates in the host vital physiological processes and development.

Alterations of the intestinal microbial populations are associated with a variety of gastrointestinal and systemic illnesses. Therefore, making attention to gut microbiota could be useful in the diagnosis and therapy procedures.

Future research studies should clarify the mechanisms that regulate the interactions between the microbiota and the host. More studies have to be done about the use of probiotics, prebiotics, and FMT in the restoration of a state of eubiosis. It is important to understand better the mechanisms of action and the duration of efficiency of the different treatments.

\section{References}

Bakken, J.S., Borody, T., Brandt, L.J., Brill, J.V., Demarco, D.C., Franzos, M.A., Kelly, C., Khoruts, A., Louie, T., Martinelli, L.P., Moore, T.A., Russell, G. and Surawicz, C. 2011. Treating Clostridium difficile infection with fecal microbiota transplantation. Clin. Gastroenterol. Hepatol. 9, 1044-1049.

Barry, K.A., Wojcicki, B.J., Middelbos, I.S., Vester, B.M., Swanson, K.S. and Fahey, G.C. 2010. Dietary cellulose, fructooligosaccharides, and pectin modify fecal protein catabolites and microbial populations in adult cats. J. Anim. Sci. 88, 2978-2987.

Bell, J.A., Kopper, J.J., Turnbull, J.A., Barbu, N.I., Murphy, A.J. and Mansfield, L.S. 2008. Ecological characterization of the colonic microbiota of normal and diarrheic dogs. Intediscip. Perspect. Infect. Dis. 2008, 17; doi:10.1155/2008/149694

Benyacoub, J., Czarnecki-Maulden, G.L., Cavadini, C., Sauthier, T., Anderson, R.E., Schiffrin, E.J. and von der Weid, T. 2003. Supplementation of food with Enterococcus faecium (SF68) stimulates immune functions in young dogs. J. Nutr. 133, 1158-1162.

Bindels, L.B., Delzenne, N.M., Cani, P.D. and Walter, J. 2015. Towards a more comprehensive concept for prebiotics. Nat. Rev. Gastroenterol. Hepatol. 12(5), 303-310.

Cammarota, G., Ianiro, G. and Gasbarrini, A. 2014. Fecal microbiota transplantation for the treatment of Clostridium difficile infection: a systematic review. J. Clin. Gastroenterol. 48(8), 693-702.

Capurso, L. 2016. I probiotici. Recenti Progressi in Medicina 107, 257-266.

Craven, M., Simpson, J.W., Ridyard, A.E. and Chandler, M.L. 2004. Canine inflammatory bowel disease: retrospective analysis of diagnosis and outcome in 80 cases (1995-2002). J. Anim. Pract. 45(7), 336-342.

Damborg, P., Sørensen, A.H. and Guardabassi, L. 2008. Monitoring of antimicrobial resistance in healthy dogs: first report of canine ampicillin-resistant 
Enterococcus faecium clonal complex 17. Vet. Microbiol. 132, 190-196.

Davies, D.R., O'Hara, A.J., Irwin, P.J. and Guilford, W.G. 2004. Successful management of histiocytic ulcerative colitis with enrofloxacin in two Boxer dogs. Aust. Vet. J. 82, 1-2.

Di Gioia, D. and Biavati, B. 2018. Probiotics and prebiotics in animal health and food safety. Springer.

FAO/WHO. 2001. Probiotics in food. Health and nutritional properties and guidelines for evaluation. Report of a joint FAO/WHO expert consultation on evaluation of health and nutritional properties of probiotics in food including powder milk with live lactic acid bacteria; Cordoba, Argentina, 2001.

Fleming, S.E., Choi, S.Y. and Fitch, M.D. 1991. Absorption of short-chain fatty acids from the rat cecum in vivo. J. Nutr. 121(11), 1787-1797.

Garcia-Mazcorro, J.F. and Minamoto, Y. 2013. Gastrointestinal microrganisms in cats and dogs: a brief review. Arch. Med. Vet. 45, 111-124.

Garcìa-Sancho, M., Rodríguez-Franco, F., Sainz, A., Mancho, C. and Rodríguez, A. 2007. Evaluation of clinical, macroscopic, and histopathologic response to treatment in non-hypoproteinemic dogs with lymphocytic-plasmacytic enteritis. J. Vet. Intern. Med. 21(1), 11-17.

Garraway, K., Johannes, C.M., Bryan, A., Peauroi, J., Rossi, G., Zhang, M., Wang, C., Allenspach, K. and Jergens, A.E. 2018. Relationship of the mucosal microbiota to gastrointestinal inflammation and small cell intestinal lymphoma in cats. J. Vet. Intern. Med. 32(5), 1692-1702.

Grønvold, A.M., L'abée-Lund, T.M., Sørum, H., Skancke, E., Yannarell, A.C. and Mackie, R.I. 2010. Changes in fecal microbiota of healthy dogs administered amoxicillin. FEMS Microbiol. Ecol. 71(2), 313-326.

Guard, B.C., Mila, H., Steiner, J.M., Mariani, C. and Suchodolski, J.S. 2017. Characterization of the fecal microbiome during neonatal and early pediatric development in puppies. PLoS One 12(4), e0175718.

Hooda, S., Vester Boler, B.M., Kerr, K.R., Dowd, S.E. and Swanson, K.S. 2013. The gut microbiome of kittens is affected by dietary protein: carbohydrate ratio and associated with blood metabolite and hormone concentrations. Br. J. Nutr. 109, 16371646.

Jergens, A.E., Schreiner, C.A., Frank, D.E., Niyo, Y., Ahrens, F.E., Eckersall, P.D. and Evans, R.A. 2003. Scoring index for disease activity in canine inflammatory bowel disease. J. Vet. Intern. Med. 17(3), 291-297.

Junhyung, K., Jae-Uk, A., Woohyun, K., Soomin, L. and Seongbeom, C. 2017. Differences in the gut microbiota of dogs (Canis lupus familiaris) fed a natural diet or a commercial feed revealed by the Illumina MiSeq platform. Gut Pathog. 9, 68.
Kelly, C.R., Kahn, S., Kashyap, P., Laine, L., Rubin, D., Atreja, A. and Wu, G. 2015. Update on fecal microbiota transplantation 2015: indications, methodologies, mechanisms, and outlook. Gastroenterology 149(1), 223-237.

Kilpinen, S., Spillmann, T., Syrjä, P., Skrzypczak, T., Louhelainen, M. and Westermarck, E. 2011. Effect of tylosin on dogs with suspected tylosin-responsive diarrhea: a placebo-controlled, randomized, doubleblinded, prospective clinical trial. Acta Vet. Scand. 53(1), 26.

Liévin-Le Moal, V. and Servin, A.L. 2006. The front line of enteric host defense against unwelcome intrusion of harmful microorganisms: mucins, antimicrobial peptides, and microbiota. Clin. Microbiol. Rev. 19, 315-337.

Lubbs, D.C., Vester, B.M., Fastinger, N.D. and Swanson, K.S. 2009. Dietary protein concentration affects intestinal microbiota of adult cats: a study using DGGE and qPCR to evaluate differences in microbial populations in the feline gastrointestinal tract. J. Anim. Physiol. Anim. Nutr. 93, 113-121.

Makielski, K., Cullen, J., O'Connor, A. and Jergens, A.E. 2018. Narrative review of therapies for chronic enteropathies in dogs and cats. J. Vet. Intern. Med. 33(1), 11-22.

Middelbos, S., Vester Boler, B.M., Qu, A., White, B.A., Swanson, K.S. and Fahey, G.C. 2010. Phylogenetic characterization of fecal microbial communities of dogs fed diets with or without supplemental dietary fiber using 454 pyrosequencing. PLoS One 5, e9768.

Minamoto, Y., Dhanani, N., Markel, M.E., Steiner, J.M. and Suchodolski, J.S. 2014. Prevalence of Clostridium perfringens, Clostridium perfringens enterotoxin and dysbiosis in fecal samples of dogs with diarrhea. Vet. Microbiol. 174, 463-473.

Münster, M., Hörauf, A. and Bilzer, T. 2006. Assessment of disease severity and outcome of dietary, antibiotic, and immunosuppressive interventions by use of the canine IBD activity index in 21 dogs with chronic inflammatory bowel disease. Berl. Munch. Tierarztl. Wochenschr. 119(11-12), 493-505.

O'Hara, A.M. and Shanahan, F. 2006. The Gut Flora As A Forgotten Organ. EMBO Rep. 7, 688-693.

Pinna, C., Vecchiato, C.G., Bolduan, C., Grandi, M., Stefanelli, C., Windisch, W., Zaghini, G. and Biagi, G. 2018. Influence of dietary protein and fructooligosaccharides on fecal fermentative endproducts, fecal bacterial populations and apparent total tract digestibility in dogs. BMC Vet. Res. 14(1), 106.

Ritchie, L.E., Steiner, J.M. and Suchodolski, J.S. 2008. Assessment of microbial diversity along the feline intestinal tract using 16S rRNA gene analysis. FEMS Microbil. Ecol. 63(3), 590-598.

Rossi, G., Pengo, G., Caldin, M., Piccionello, A.P., Steiner, J.M., Cohen, N.D. and Suchodolski, J.S. 
2014. Comparison of microbiological, histological, and immunomodulatory parameters in response to treatment with either combination therapy with prednisone and metronidazole or probiotic VSL\#3 strains in dogs with idiopathic inflammatory bowel disease. PLoS One 9(4), e94699.

Sanders, M.E., Merenstein, D., Merrifield, C.A. and Hutkins, R. 2018. Probiotics for human use. Nutr. Bull. 43(3), 212-225.

Sauter, S.N., Allenspach, K., Gaschen, F., Gröne, A., Ontsouka, E. and Blum, J.W. 2005. Cytokine expression in an ex vivo culture system of duodenal samples from dogs with chronic enteropathies: modulation by probiotic bacteria. Domest. Anim. Endocrinol. 29(4), 605-622.

Savage, D.C. 1977. Microbial ecology of the gastrointestinal tract. Annu. Rev. Microbiol. 31, 107-133.

Schmitz, S. and Suchodolski, J. 2016. Understanding the canine intestinal microbiota and its modification by pro-, pre- and synbiotics - what is the evidence? Vet. Med. Sci. 2(2), 71-94.

Simpson, J.M., Martineau, B., Jones, W.E. Ballam, J.M. and Mackie, R.I. 2002. Characterization of fecal bacterial populations in canines: effects of age, breed and dietary fiber. Microbiol. Ecol. 44, 186-197.

Simpson, K.W. 2011. Managing chronic enteropathies in dogs, small animal and exotics. In Proceedings of the North American Veterinary Conference Orlando, Florida, USA, 15th-19th January, 2011, pp: 650-656.

Staggers, J.E., Frost, S.C. and Wells, M.A. 1982. Studies on fat digestion, absorption, and transport in the suckling rat. III. Composition of bile and evidence for enterohepatic circulation of bile salts. J. Lipid Res. 23(8), 1143-1151.

Steiner, J.M. and Ruaux, C.G. 2008. Laboratory tests for the diagnosis of intestinal disorders. Ed.
Small animal gastroenterol. Hanover, Germany: Schluetersche, pp: 50-55.

Suchodolski, J.S. 2011. Intestinal microbiota of dogs and cats: a bigger world than we thought. Vet. Clin. North Am. Small Anim. Pract. 41, 261-272.

Suchodolski, J.S. and Simpson, K. 2013. Canine gastrointestinal microbiome in health and disease. Vet. Focus 23(2), 22.

Suchodolski, J.S., Dowd, S.E., Wilke, V., Steiner, J.M. and Jergens, A.E. 2012. 16S rRNA gene pyrosequencing reveals bacterial dysbiosis in the duodenum of dogs with idiopathic inflammatory bowel disease. PLoS One 7, e39333.

Weese, J.S., Staempfli, H.R., Prescott, J.F., Kruth, S.A., Greenwood, S.J. and Weese, H.E. 2001. The roles of Clostridium difficile and enterotoxigenic Clostridium perfringens in diarrhea in dogs. J. Vet. Intern. Med. 15, 374-378.

Westermarck, E., Skrzypczak, T., Harmoinen, J., Steiner, J.M., Ruaux, C.G., Williams, D.A. and Rinkinen, M. 2005. Tylosin-Responsive Chronic Diarrhea in Dogs. J. Vet. Intern. Med. 19(2), 177186.

Wong, J.M., De, S.R., Kendall, C.W., Emam, A. and Jenkins, D.J. 2006. Colonic health: fermentation and short chain fatty acids. J. Clin. Gastroenterol. 40, 235-243.

Xenoulis, P.G., Palculict, B., Allenspach, K., Steiner, J.M., Van House, A.M. and Suchodolski, J.S. 2008. Molecular-phylogenetic characterization of microbial communities' imbalances in the small intestine of dogs with inflammatory bowel disease. FEMS Microbiol. Ecol. 66, 579-589.

Zentek, J., Marquart, B., Pietrzak, T., Ballèvre, O. and Rochat, F. 2003. Dietary effects on bifidobacteria and Clostridium perfringens in the canine intestinal tract. J. Anim. Physiol. Anim. Nutr. 87, 397-407. 